\title{
A Study of Hematological Changes in Neonatal Sepsis
}

\author{
Khushbu Dalasukhbhai Makadia and Pragnesh Shah*
}

Department of Pathology, Govt. Medical College, Bhavnagar, Gujarat, India.

\begin{abstract}
Background: Neonatal sepsis is one of the major causes of morbidity and mortality in the newborn. Early diagnosis and treatment are crucial in the management of neonatal sepsis. Blood culture, the gold standard, takes long time and lack sensitivity. Hematological sepsis score is a simple score obtained by combining routinely available tests and is rapid, non-expensive and has shown good correlation with blood culture. With this background the study was carried out to evaluate hematological changes in neonatal sepsis and to analyse the diagnostic utility of hematological scoring system (HSS) and its correlation with C- reactive protein and blood culture in neonatal sepsis.

Methods: The study included 200 neonates admitted to Neonatal Intensive Care Unit with clinical suspicion of neonatal sepsis. CBC, Peripheral blood smear, CRP and Blood culture were obtained from all the neonate. Hematological sepsis score was calculated, and its sensitivity and specificity were obtain using blood culture as gold standard.
\end{abstract}

Result: We screened 200 neonates with suspected neonatal sepsis. Blood culture was positive in $101(50.5 \%)$ and HSS score $>2$ was found in $80(40 \%)$ neonates. CRP and HSS had sensitivity of $84.15 \%$ and $72.27 \%$ and specificity of $68.68 \%$ and $92.92 \%$ respectively.

Conclusion: Our study shows that HSS had fairly good sensitivity and specificity for neonatal sepsis screening and HHS can be utilized for neonatal sepsis screening.

Keywords: Neonatal Sepsis, Hematological Scoring System, Blood Culture, C-Reactive Protein.

\section{Introduction}

Neonatal sepsis is defined by systemic infections in first 28 days of life. ${ }^{[1]}$ Preterm birth complications and infections are the largest contributors to the neonatal mortality. The incidence of neonatal sepsis according to the data from National Neonatal Perinatal Database (NNPD, 2002-03) is 30 per 1000 live births. Mortality due to sepsis can be prevented by early diagnosis, rational use of antibiotics and aggressive supportive care. However, early recognition is difficult as the sign and symptoms of early sepsis are non-specific. Blood culture is regarded as the gold standard test, but it takes around 48-72 hours. Further yield of blood culture is $30-70 \%$, so some neonates may be missed. ${ }^{[2]}$ Furthermore, inability to exclude sepsis early results in the unnecessary exposure to antibiotics to the infants who do not have sepsis. Novel markers, like Interleukin-6, Interlukin-8, plasma elastase, are more sensitive early diagnosis but are not routinely available and are impractical for use in developing country like India. Leukopenia, toxic granules, immature neutrophil to total neutrophil ratio, thrombocytopenia, micro-ESR, C-reactive protein are some of the indirect markers of sepsis. These investigations are collectively referred to as sepsis screen. These investigations are rapid and available routinely. Presence of two or more of these in the background of clinical suspicion is positive sepsis screen. These can be useful for early recognition of sepsis and guide in treatment initiation. These are particularly useful in the resource limited setting where advance markers are not available. The current study is being undertaken therefore to study hematological changes in the neonatal sepsis and to analyse the diagnostic utility of hematological scoring system (HSS) and its correlation with $\mathrm{C}$ - reactive protein and blood culture in neonatal sepsis.

\section{Material and Method}

The study was conducted in tertiary care teaching hospital in Bhavnagar, Gujarat, India from $1^{\text {st }}$ April 2018 to $1^{\text {st }}$ April 2019. Ethical approval from institutional ethical committee was obtained and written and informed consent was obtained from the parents. A total of 200 neonates admitted with clinically suspected septicemia were included in the study. Neonates who received antibiotics before admission, underwent surgery, had congenital anomalies or inborn errors of metabolism and neonates of mother with pregnancy induced hypertension were excluded. A detailed history of basic demographic data like age, gender, gestational age, mode of delivery, birth weight was recorded. Clinical features at the time of admission to hospital were obtained. These included refusals to feed fever, hypothermia, respiratory distress, abdominal pain, pallor, jaundice, convulsion, lethargy and rash. Blood 
counts were done using automated hematological analyser and also manually by examination of stained slides. Automated hematological analyser utilised was Nihon kohden. Slides were prepared by using the field stain. Total WBC count, absolute neutrophil count, immature to mature neutrophil ratio (I:M ratio), degenerative change in PMN, Platelet count, C-reactive protein (CRP) and blood culture were obtained. The cut-off values of above blood tests are mentioned in the table 1 and were taken from Manroe BL et al. ${ }^{[3]}$ Blood culture were incubated for 48 hours before labelling them as negative. CRP estimation was done by latex agglutination method. Hematological scoring of Rodwell (HSS) was obtained as per table 1. HSS was interpreted as sepsis unlikely if $<2$, sepsis possible if between 3 and 4, and sepsis very likely if $>4$. Data was tabulated in Microsoft Office Excel worksheet and descriptive statistics were given as means, median and standard deviations at 95\% confidence interval. Sensitivity, specificity, positive predictive value and negative predictive value of septic screen was calculated with culture outcome (gold standard). Comparison of neonates with blood culture positive and negative patients was done using Mann-Whitney U Test for continuous parameters (Weight, Age, Total leucocyte cunt, ANC, Platelet count). Comparison of neonates with blood culture positive and negative neonates was done using Fischer's exact test for non-continuous variables (Gender). A p value less than 0.05 was considered as significant at $95 \%$ confidence interval.

\section{Results}

A total 200 neonates with $112(56 \%)$ male were included in the study. Mean age was 7.37 days and mean birth weight was $1.84 \mathrm{~kg}$ with $80 \%$ of total neonates having low birth weight. Blood cultures were positive in $101(50.5 \%)$ of neonates. Most common organism were Klebsiella, followed by E. Coli and Acinetobacter. Frequency of identified organism is shown in the figure 1 . Table 2 shows comparison of blood culture positive and negative neonates. Blood culture positive neonates were more likely to have prematurity, low birth weight, raised CRP, I:T and $\mathrm{I}: \mathrm{M}$ ratio, raised total $\mathrm{PMN}$ and $\mathrm{WBC}$ counts and a higher HSS score.

A total of 80 neonates had Haematological sepsis score of Rodwell of $>2$. HSS $>2$ had sensitivity of $72.27 \%$ and specificity of $92.92 \%$. Positive and negative predictive value were $91.25 \%$ and $76.66 \%$. As compared to individual components combined HSS of $>2$ had higher sensitivity, except for I:T PMN ratio. Similarly, specificity was also high for HSS except for immature PMN counts and I:M PMN ratio. Table 3 summaries sensitivity, specificity, positive and negative predictive values of HSS and subcomponent of HSS. Taking HSS $>3$ as positive sepsis screen sensitivity was decreased to $55.44 \%$ however, specificity was increased to $98.98 \%$. Similarly, HSS $>4$ had sensitivity $45.54 \%$ and specificity of $100 \%$. Positive and negative predictive values of HSS $>4$ were $100 \%$ and $64.28 \%$ respectively. Sensitivity of CRP and HSS $>2$ was $73.27 \%$ and $72.27 \%$ respectively considering blood culture as gold standard. Specificity was $80.95 \%$ and $92.92 \%$ respectively.

\section{Discussion}

Neonatal sepsis is a common cause of neonatal mortality. Outcome depends on early diagnosis and initiation of

Table 1: Hematological scoring system of Rodwell.

\begin{tabular}{|l|l|l|}
\hline \multicolumn{1}{|l}{ Abnormality } & Score \\
\hline I;T ratio & Increased (normal is 0.16 in first 24 hours of life and 0.12 after 24 hours of life) & 1 \\
\hline Total PMN count & $\begin{array}{l}\text { Increased or decreased (normal range: first } 60 \text { hours } 7800-14500,60-120 \text { hours } \\
1800-7000,120 \text { hours onward } 1800-5400) \\
\text { No mature PMN seen }\end{array}$ & 1 \\
\hline I:M ratio & $\geq 0.3$ & 2 \\
\hline Immature PMN count & $\begin{array}{l}\text { Increased (Normal is }<1400 / \mathrm{mm}^{3} \text { in first } 24 \text { hours, }<1000 / \mathrm{mm}^{3} \text { in } 24-48 \text { hours and } \\
<600 / \mathrm{mm}^{3} \text { in } 48-60,<500 / \mathrm{mm}^{3} \text { after } 60 \text { hours) }\end{array}$ & 1 \\
\hline Total WBC count & $\begin{array}{l}\leq 5000 / \mathrm{mm}^{3} \text { or } \geq 25000 / \mathrm{mm}^{3} 30,000, \text { and } \\
21,000 / \mathrm{mm}^{3} \text { at birth, } 12-24 \mathrm{~h}, \text { and day } 2 \text { onward, respectively) }\end{array}$ & 1 \\
\hline Degenerative change in PMN & $\geq 3+$ for vacuolization, toxic granulation, or Dohle bodies & 1 \\
\hline Platelet count & $\leq 150000 / \mathrm{mm}^{3}$ & 1 \\
\hline
\end{tabular}

I:T immature to total neutrophil count, I:M immature to mature neutrophil count, PMN-polymorphonuclear cell. Normal value taken from Manroe BL et al. [II] 
Table 2: Comparison of blood culture positive and negative neonates.

\begin{tabular}{|l|l|l|l|}
\hline Characteristics & Blood culture positive & Blood culture negative & P value \\
\hline Age (mean \pm SD) & $6.83 \pm 4.00$ & $7.29 \pm 4.30$ & 0.073 \\
\hline Gender (male) & 44 & 54 & 0.157 \\
\hline Birth weight (mean \pm SD) $\mathrm{Kg}$ & $1.55 \pm 0.44$ & $2.16 \pm 0.59$ & 0.00001 \\
\hline Total Leucocyte count cells $/ \mathrm{mm}^{3}($ mean \pm SD) & $12077 \pm 7740$ & $6294 \pm 1896$ & $P<0.00001$ \\
\hline ANC (mean \pm SD) cells $/ \mathrm{mm}^{3}$ & $7409.5 \pm 5163$ & $3168 \pm 1225$ & $\mathrm{p}<0.00001$ \\
\hline Degenerative changes in neutrophils (mean \pm SD) & $4.32 \pm 3.02$ & $1.24 \pm 0.55$ & $\mathrm{p}<0.00001$ \\
\hline I:T PMN ratio (mean \pm SD) & $0.18 \pm 0.09$ & $0.08 \pm 0.07$ & $\mathrm{p}<0.00001$ \\
\hline I:M PMN ratio (mean \pm SD) & $0.25 \pm 0.20$ & $0.10 \pm 0.17$ & $p<0.00001$ \\
\hline Platelet count (mean \pm SD) & $1.59 \pm 1.01$ & $2.38 \pm 0.65$ & $p<0.00001$ \\
\hline HSS (mean \pm SD) & $3.90 \pm 1.80$ & $0.82 \pm 0.95$ & $P<0.00001$ \\
\hline
\end{tabular}

Table 3: Sensitivity, specificity, positive and negative predictive value of HSS and subcomponent of HSS.

\begin{tabular}{|l|l|l|l|l|}
\hline Parameter & Sensitivity & Specificity & PPV & NPV \\
\hline I:T PMN ratio & $85.14 \%$ & $82.82 \%$ & $83.49 \%$ & $84.53 \%$ \\
\hline Total PMN & $64.35 \%$ & $74.74 \%$ & $72.22 \%$ & $67.27 \%$ \\
\hline I:M PMN ratio & $25.74 \%$ & $97.97 \%$ & $92.85 \%$ & $56.39 \%$ \\
\hline Immature PMN & $55.44 \%$ & $97.97 \%$ & $96.55 \%$ & $68.30 \%$ \\
\hline Total WBC & $48.51 \%$ & $71.71 \%$ & $63.63 \%$ & $57.72 \%$ \\
\hline HSS $>\mathbf{2}$ & $72.27 \%$ & $92.92 \%$ & $91.25 \%$ & $76.66 \%$ \\
\hline
\end{tabular}

Table 4: Diagnostic accuracy of HSS.

\begin{tabular}{|c|l|l|l|}
\hline S. No & Study & Sensitivity (\%) & Specificity (\%) \\
\hline 1 & Mondal SK et al [3] & 84 & 84 \\
\hline 2. & Sriram et al. [12] & 55.30 & 91.7 \\
\hline 3 & Misra RN et al [13] & 100 & 37.5 \\
\hline 4 & Bhale CP et al [8] & 93.4 & 77.0 \\
\hline 5 & Vinay BS et al. [7] & 77 & 41 \\
\hline 6 & Present study & 72.27 & 92.92 \\
\hline
\end{tabular}

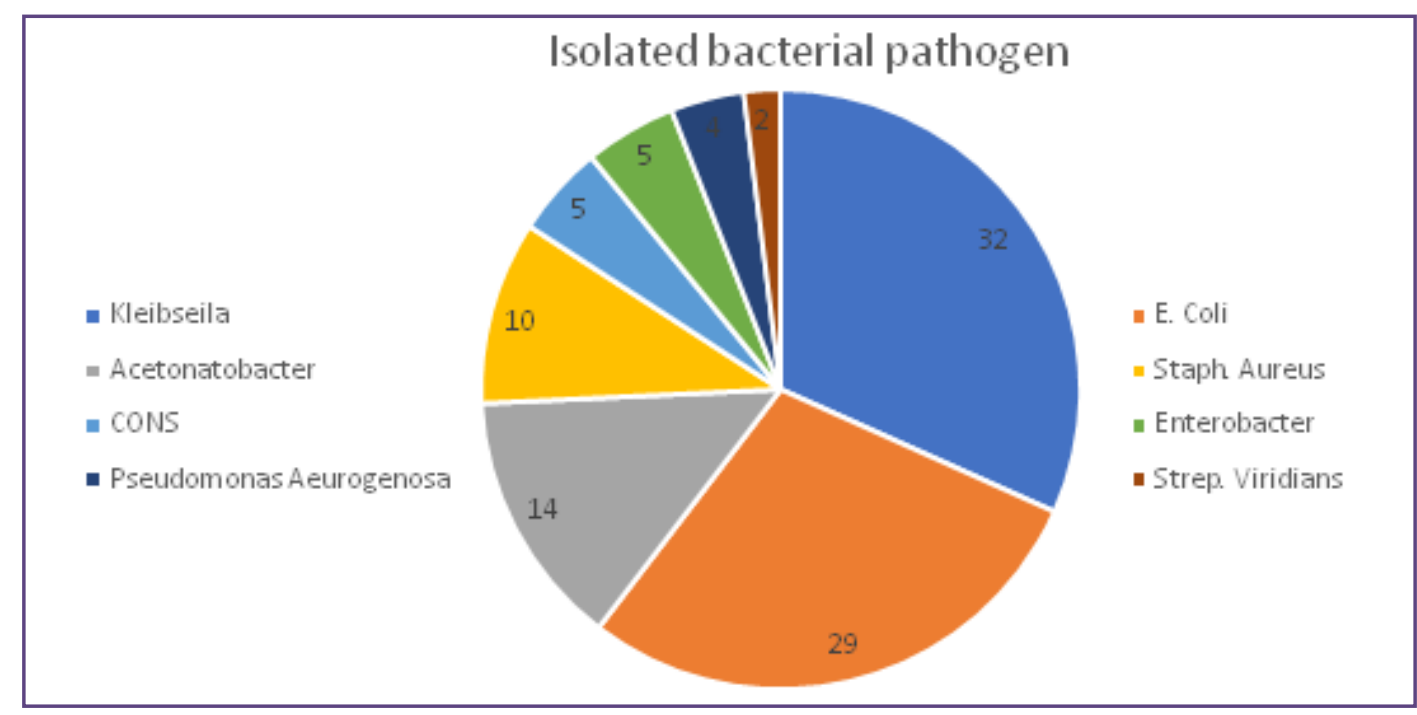

Fig. 1: Frequency of isolates in blood culture. 
appropriate antibiotics. Traditional method for diagnosis of neonatal sepsis is blood culture. However, blood culture has sensitivity of $50-70 \%$ and results take at least 48 hours. Alternative methods for diagnosis include inflammatory markers, cytokines and molecular markers. Despite being sensitive and showing changes early in the course of neonatal sepsis these markers are not utilised routinely in view of cost and limited availability. Hematological changes in neonatal sepsis includes leucocytosis or leucopoenia, increase or decrease in ANC, increase in immature neutrophils and change in immature to mature PMN ratio. However, individual hematological change lack enough sensitivity or specificity. Haematological sepsis score of Rodwell combines various hematological changes and increases sensitivity and specificity. Our study included 200 neonates with suspected neonatal sepsis. Blood culture was positive in $101(50.5 \%)$ of patients. Considering blood culture as gold standard HSS $>2$ had sensitivity of $72.27 \%$ and specificity of $92.92 \%$.

\section{Diagnostic Accuracy of CRP}

CRP had sensitivity $84.15 \%$ and specificity was $68.68 \%$. Positive and negative predictive value was $73.27 \%$ and $80.95 \%$ respectively. Other studies also have shown similar sensitivity and -specificity ranging from $81-90 \%$ and 50 $84 \%$ respectively. ${ }^{[3][4][5][6][7][8]}$

\section{Diagnostic Accuracy of Total WBC Counts}

Total WBC counts had sensitivity of $64.35 \%$ and specificity of $74.74 \%$. Previous studies also have shown variable results with sensitivity ranging from $43-75 \%$ and specificity ranging from $74-94 \%$. Studies have shown wide variability in the sensitivity ranging from $43-75 \%$ and specificity ranging from $74-94 \% .{ }^{[4][6][8][9][10]}$

\section{Diagnostic accuracy of total ANC}

In present study, ANC had sensitivity of $64.35 \%$ and specificity of $74.74 \%$. Other studies have shown sensitivity ranging from $42-80 \%$ and specificity ranging from 79 $99 \%{ }^{[6][8][9][10]}$

\section{Diagnostic accuracy of I:M PMN ratio}

In present study I:M PMN ratio had lower sensitivity $25.74 \%$ but higher specificity of $97.97 \%$. This contrasts with other studies wherein sensitivity was high. ${ }^{[6][10]}$ However, Makkar M. et al reported low sensitivity and high specificity. ${ }^{[9]}$

\section{Diagnostic accuracy of I:T PMN ratio}

In present study I:T PMN ratio had sensitivity of $85.14 \%$ and specificity of $82.82 \%$. Other studies have shown variable results. Makkar $\mathrm{M}$ et al had high sensitivity and specificity whereas Vandana $G$ et al had low sensitivity and specificity. ${ }^{[5][9]}$

\section{Diagnostic accuracy of HSS of Rodwell}

In present study $\mathrm{HHS}>2$ had sensitivity of $72.27 \%$ and specificity of $92.92 \%$. HSS $>2$ has sensitivity ranging from $55-100 \%$ while specificity was $37-91 \%$. Misra RN et al, reported very high sensitivity but low specificity, whereas Mondal SK et al, had similar sensitivity and specificity as our study. Table 4 summarise diagnostic performance of HSS in various studies.

\section{Conclusion}

Neonatal sepsis is common cause of neonatal mortality. Early diagnosis and timely initiation of antibiotics is vital for effective management. HSS is an easy, cost-effective and can be obtained by test done routinely. Our study showed HSS has a sensitivity of $72.27 \%$ and specificity of $92.92 \%$ as compared to blood culture. So HSS can be used for the screening of neonatal sepsis.

\section{Funding}

No funding was received for this study

\section{Competing Interests}

There are no conflict of interest.

\section{References}

1. Chaurasia S, Sivananda S, Agarwal R, Ellis S, Sharland M, Sankar MJ. Neonatal sepsis in South Asia: huge burden and spiralling antimicrobial resistance. BMJ 2019;364:K5314.

2. Gengaimuthu K, Karthikeyan V. Towards an ideal neonatal sepsis screen panel - A review Karthikeyan. Indian J. Child Health 2017;4:614-8.

3. Mondal SK, Nag DR, Bandyopadhyay R. Neonatal sepsis : Role of a battery of immunohematological tests in early diagnosis. Int. J. Appl. Basic Med. Res. 2012;2:43-8.

4. Pal K, Samanta AK. Evaluation OfHematological Parameters In Early Onset Neonatal Sepsis. NJIRM 2013;4:29-34.

5. Vandana G, Lokesh S, Kavita B. Haematological Profile in Neonatal Septiceamia. IOSR J. Dent. Med. Sci. 2017;16:11-7.

6. Ms S, Alva SR, Vs S, Tm K. Research article evaluation of neonatal septicaemia using hematological parantelers. Int. J. Recent Sci. Res. 2015;6:2775-8.

7. Bs V, Girish GN, Adhikari S, Hugara S. Evaluation of Septic Screen as a Diagnostic Tool for Neonatal Sepsis in a Tertiary Hospital at Mysore. Sch. J. Appl. Med. Sci. 2015;3:1005-10.

8. Bhale CP, Kale AV, Kale SS, Mahajan M, Smulay S. Utility of Sepsis Screen in the Early Diagnosis of Neonatal Sepsis. Indian J. Neonatal Med. Res. 2016;4:1-7.

9. Makkar M, Gupta C, Pathak R, Garg S, Mahajan NC. Performance Evaluation of Hematologic Scoring System in Early Diagnosis of Neonatal Sepsis. J. Clin. Neonatol. 2013;2:25-9. 
10. Bhalodia M, Surekha B, Hippargi M, Patil M. Role of Hematological Scoring System in Diagnosis of Neonatal Sepsis. J. Clin. Neonatol. 2017;6:1-4.

11. Manroe BL, Weinberg AG, Rosenfield CR, Browne $\mathrm{R}$. The neonatal blood count in health and disease . L Reference values for neutrophilic cells. Fetal Neonatal Med. 1979;95:89-98.
12. Sriram R. Correlation of Blood culture results with the Sepsis score and the Sepsis screen in the diagnosis of Neonatal Septicemia . Int J Biol Med Res 2011;2:360-8.

13. Misra RN, Jadhav S V, Ghosh P, Gandham N, Angadi K. Role of sepsis screen in the diagnosis of neonatal sepsis. Med. J. Dr. D.Y. Patil Univ. 2013;6:254-8.

*Corresponding author:

Dr. Pragnesh Shah, Professor, Department of Pathology, Sir TakhatsinhjiHospital, Govt. Medical College, Bhavnagar, Gujarat, India-364001

Phone: +919428106604

Email: drphshah73@yahoo.com

Financial or other Competing Interests: None. 